\title{
Beiträge aus dem Kirchenslavischen zu den neutestamentlichen Apokryphen und der altchrist- lichen Literatur.
}

Von Dr. Ivan Franko in Lemberg.

\section{Das Martyrium der heiligen Photine.}

Dieses Apokryphum ist kein regelrechtes Apokryphum; in keinem Apokryphenindex wird es erwähnt und destoweniger verooten. Nur das totale Vergessen seiner Urgestalt scheint es als Apokryphum zu stempeln. Zwar figuriert die heilige Photine mit ihrer zahlreichen Sippschaft sowohl in griechischen Synaxarien, als auch in lateinischen Martyrologien unter dem 2. März als Opfer der neronischen Christenverfolgung, und kurze Erzählungen von ihrem Tode sind aus griechischen Synaxarien und späteren lateinischen Legendarien bei den Bollandisten gesammelt und sogar zum Gegenstand eines gelehrten Kommentars gemacht worden. ${ }^{x}$ Doch haben die gelehrten Herausgeber eine ausführliche Fassung dieser Passion, den sogenannten Menäen-Text, entweder gar nicht $z u$ Gesicht bekommen, oder vollständig außer acht gelassen; dieses letztere mutmaße ich nur darum, weil sie auch die kurzen, farblosen Synaxartexte nur mit einigem Kopfschütteln in ihr großes Corpus aufnehmen. Doch scheint es mir wahrscheinlicher anzunehmen, der ausführliche Menäentext sei. ihnen gänzlich unbekannt geblieben, da sie sonst mit den farb- und inhaltslosen Synaxartexten, welche sich als sehr unvollkommene Inhaltsangaben' der Menäen-Passion entpuppen, nicht so viel Wesens gemacht hätten, um gelehrte Forschungen (welche obendrein zu keinem wissenschaftlich irgendwie brauchbarem Resultat führen) an sie zu knüpfen.

I Acta Sanctorum Martii tomus tertius. Parisiis et Romae 1865, pag. So-82; griechische Synaxartexte s. Propylaeum ad Acta Sanctorum Novembris. Synaxarium eccl. Constantinopolitanae e cod. Sirmondiano, nunc Berolinensi ed. Hippolytus Delahaye. Bruxelles 1902, p. 549-552. 
Um nun diesen Ausgangspunkt meiner heutigen Mitteilung gehörig zu beleuchten, sei es mir erlaubt, unter Verweisung auf die in den AASS. abgedruckten lateinischen und griechischen Texte der Passion der heiligen Photine hier eine wortgetreue Übersetzung der kirchenslavischen SynaxarPassion mitzuteilen, welche sich in kirchenslavischen Prologen auch unterm 20. März findet und von mir aus einem 1632 geschriebenen Prolog in Bd. II meiner Denkmäler (S. 367) abgedruckt wurde. ${ }^{x}$

Passion der heiligen Märtyrerin Phetynia der Samáriterin und ihres Sohnes Josephus. Phetynia, die berühmte Samariterin, mit welcher der Herr am Brunnen sich unterredete, siè kam auch während der Regierungszeit Neros von Samarien nach Rom, um die heiligen Apostel Petrus und Paulus zu begrüßen. Hernach wohnte sie mit ihrem Sohne Josias in der Stadt Karthago, Christus als den wahren Gott predigend. Ihr Sohn aber wurde ein tapferer Krieger, kämpfte gegen die Barbaren und wurde ein Heerführer. Und er wurde vom Kaiser nach Gallien geschickt, um alle dort lebenden Christen zu töten. $\mathrm{Da}$ er sie aber nicht tötete, sondern im Gegenteil alle lehrte Christen zu werden und viele zum Glauben bekehrte, wie er auch den Dux Sebastian selbst dazu brachte an Christus zu glauben, befahl der Kaiser, nachdem er dies erfahren hatte, ihn $z u$ fesseln und zu sich zu bringen mit andern, welche verschiedentlich gemartert wurden. Den einen wurden die Augen ausgebohrt, die andern aber wurden in einen von giftigen Schlangen gefüllten Kerker hineingeworfen. Hier aber erschien ihnen der Herr mit Petrus und Paulus und stärkte sie. Und nach drei Jahren .führte er (scil. Nero) ihn heraus und hängte ihn kopfunter an einen Baum und alle die bei ihm befindlichen Gläubigen und kratzte sie mit eisernen Nägeln, hernach wurde allen die Haut abgeschunden und den Männern wurden die Schamteile abgeschnitten und den Hunden vorgeworfen. Die heilige Phetynia aber, nachdem sie geschunden worden, wurde an die Wipfel der zwei zusammengebogenen Bäume gebunden, damit sie entzweigerissen werde, Gott aber beschützte sie davor. Alle übrigen starben vom Schwert, die heilige Phetynia aber lebte noch viele Tage im Kerker und Gott dankend entschlief sie im Frieden.

Es lohnt nicht der Mühe, die geringfügigen Abweichungen dieses kirchenslavischen Textes von den in den AASS. veröffentlichten griechischen und lateinischen Texten zu notieren, sobald wir wissen, daß alle

x Monumenta linguae nec non litterarum ukraino-russicarum, vol. II. Codex apocryphus, part II. Evangelia apokrypha (Leopolis I899, p. 767. 
diese Texte nur Kürzungen einer ausführlichen Passion sind. Da ich keine griechischen Menäen zur Hand habe, kann ich nicht ausdrücklich sagen, ob sich diescr ausführliche Passionstext auch dort befindet; jedenfalls muß es handschriftliche griechische Menäen geben, in denen er zu finden sein wird, da ja der kirchenslavische Text, auf wclchen ich glcich zu sprechen kommc, gewif cine Übersetzung aus dem griechischen ist. Dieser kirchenslavische Text wurde vielfach gedruckt, zuerst im Moskaucr Prologus 1685, und ist auch in Handschriften verbreitet. Ich habe ihn in Lemberg in zwei Kopien gefunden: die eine in einem kirchenslavischen Menäon aus dem Jahre 1492 (in Jassy geschrieben), dic andere in einer neueren (Ende des XVI. und Anfang des XVII. Jahrhunderts) in Lemberg geschriebenen griechisch-russischen Handschrift; beide Handschriften befinden sich in der Ossolinski-Bibliothek unter den NN. 368 und 827. Der Text der älteren Kopie wurde von mir im dritten Bande meines Codex apocryphus (Monumenta III, p. 364-369) herausgegeben, woraus ich hier eine wortgetreue Übersetzung mitteile.

An demselben Tage (20. März) das Andenken der heiligen Märtyrerin Christi Photine der Samariterin, mit welcher Christus beim Brunnen sprach, und der mit ihr Gemarterten.

In den Tagen des römischen Kaisers Nero wurde eine grobe Christenverfolgung erhoben. Nach dem Martyrium der heiligen Apostelhäupter Petrus und Paulus wurden ihre Jünger fleibig gesucht. Damals lebte im afrikanischen Karthago die heilige Photine mit ihrem Sohne Josias und verkündigte mutig das Evangelium Christi. Ihr älterer Sohn Viktor aber hatte viele Heldentaten vollbracht in dem Kriege gegen die Avaren, welche die Römer bekriegten, und wurde hernach als Heerführer vom Kaiser nach Italien geschickt, um alle dort anwesenden Christen zu martern. Als aber Dux Sebastian dies hörte, sagte er: „Ich weiß es sicher, o Heerführer ${ }^{x}$, daß du ein Christ bist und daß deine Mutter mit deinem Bruder Josias dem Petrus nachfolgen. Du aber erfülle den Befehl des Kaisers, damit du nicht mit der Seele verloren gehst."

Und Viktor sprach: „Ich will den Willen des himmlischen Kaisers und unsterblichen Gottes erfüllen, den Befehl aber des Kaisers Nero in betreff der Marterung der Christen achte ich als nichts." "

Dux aber antwortete: „Als meinem herzlichen Freunde habe ich dir

In Slavischen rojevoda, General.

2 Slavisch: ne bregú - vernachlässige, mißachte ich. 
diesen Rat gegeben. Denn wenn du neben dem Wege aufzulauern beginnst und die vorbeigehenden Christen ausfragst und marterst, wirst du sowohl dem Kaiser Angenehmes tun, als auch dich selbst mit christlicher Habe bereichern. Erkläre aber auch brieflich deiner Mutter und deinem Bruder, daß sie nicht so mutig die Griechen ihren väterlichen Kultus zu verlassen lehren, damit auch du ihretwegen nicht gleich ihnen leidest. Insgeheim aber möget ihr euren Glauben an Christum behalten, wie ihr wollt."

Viktor aber sprach: „Fern sei mir solches zu tun, daß ich Christen martere oder irgend etwas von ihnen nehme odér meiner Mutter und meinem Bruder den Rat gebe, nicht żu predigen, Christus sei ein Gott. Im Gegenteil, auch ich selbst bin ein Verkünder Christi, bin und werde sein wie sie, und wir werden alle das Übel erfahren, das da kommen soll."

Dux aber sprach: „Ich habe dir Nützliches gesagt, du aber wirst selbst sehen, was kommen wird."

Und nachdem er dies gesagt hatte, stieg ihm ein Brand im Antlitz auf und er fiel $z u$ Boden vor heftigem und großen und scharfen Augenleiden, und konnte nichts mehr sagen. ${ }^{x}$ Die Anwesenden aber hoben ihn auf und legten ihn ins Bett. Und er lag da drei Tage ohne ein Wort gesagt $z u$ haben, nach dem dritten Tage aber schrie er mit großer Stimme und rief: „Groß ist der christliche Gott!"s

Da kam Viktor zu ihm herein und sprach: „Warum hast du dich so plötzlich verändert?“

Er aber sprach: "Christus ruft mich, süßester Viktor."

Und er taufte sich gleich, von Viktor belehrt, und als er aus dem Wasser herauskam, gab er plötzlich Gott die Ehre." Und als das Volk dieses ruhmvolle Wunder sah, bekam es Furcht, es möge, im Unglauben verharrend, von ebensolchem Leiden heimgesucht werden, und es kam herbei und taufte sich.

Hernach aber kam es zu Neros Gehör, daß Viktor, der italische Heerführer, und Sebastian, der Dux derselben Stadt, die Predigt des Petrus und Paulus verkündigen und alle zum Christo führen, andererseits aber auch die Mutter des Stratelates Photine mit ihrem Sohn Josias in Karthago nach einem Brief dasselbe tun. Nachdem der Kaiser dies gehört hatte, entbrannte er vor Zorn und schickte Krieger nach Italien,

I Wörtlich slavisch: und verweilte stimmlos.

2 D. h. er wurde gesund. 
um dic dort weilenden Christen, Männer und Weiber herbeizuführen. Ihnen allen aber crschien Christus und sagte: „Kommt zu mir alle Mühevollen und Beladenen, und ich werde euch zur Ruhe bringen. Denn ich bin mit euch, und Nero und scinc Gefolgschaft werden besiegt werden." Und dann sprach er zu Viktor: "Von nun an wird Photinus dein Name sein. Denn viele wcrden durch dich erleuchtet zu mir kommen. Sebastian aber, sofern er durch das Wort zum Martyrium gestïrkt wird, selig wird er scin, der bis ans Ende ausharrt." Nachdem der Herr dies gesprochen, stieg er in den Himmel hinauf.

Der heiligen Photine offenbarte er auch alles, was geschehen sollte, und sie hob sich auf von Karthago mit vielen Christen und kam in das große Rom. Und die ganze Stadt geriet in Bewegung ${ }^{x}$ und sprach: "Wer ist diese?" Sie aber verkündigte mutig den Namen Christi. Da wurde auch ihr Sohn Photinus mit dem Dux Sebastianus und den Soldaten herbeigefuihrt. Doch die heilige Photine kam ihm zuvor und stellte sich vor Nero mit Josias und ihrem Anhang. ${ }^{2}$ Und Nero sprach zu ihr: „Weswegen kamt ihr zu uns?" Die heilige Photine aber sprach: „Um dich zu lehren Christum zu verehren." Da sagten zum Kaiser die Wachehaltenden: „Dux Sebastian und der Heerführer Viktor, welche an die Götter nicht glauben, sind aus Italien angekommen." Und der Kaiser sprach: "Sie sollen vorgeführt werden."

Als diese vorgeführt wurden, sprach der Kaiser zu ihnen: „Was ist's, was ich von euch gehört habe?"

Die Heiligen sprachen: „Was du von uns gehört hast, o Kaiser, ist wahr."

Nero aber blickte auf die heiligen Frauen und sprach zu ihnen: „Wollt ihr auch euch von Christus absagen, oder zieht ihr es vor, eines schlimmen Todes zu sterben? Wer seid ihr und wie nennt ihr euch?“

Die heilige [Photine] aber sprach: „Ich wurde vom Christus, meinem Gott, Photine genannt. Meine Schwestern aber, die mit mir sind, heißen die erste Anatole, die zweite Photo, die dritte Photis, die vierte Parasceve, die fünfte Kyriake. Meine Söhne aber sind der erste Viktor, der von dem Herm Photinus genannt wurde, der zweite aber Osis."

Und Nero sprach: „Seid ihr alle einig darin, gemartert $z u$ werden und im Nasoräer Christus zu sterben?"

Die Heilige sprach: „Seinetwegen zu sterben sind wir alle mit Freude und Lust übereingekommen."

I Slavisch: protresé se - erzitterte.

2 Wörtlich: und den mit ihr. 
Dann befahl der Kaiser, mit eisernen Hämmern die Glieder ihrer Finger zu zermalmen. $\mathrm{Da}$ wurde ein Amboß gebracht, und die Heiligen legten ihre Hände darauf, und die Diener begannen draufzuschlagen. Und von der dritten Stunde bis zur neunten wechselten dreimal die Schlagenden, die Heiligen aber fühlten gar keinen Schmerz. Als Nero dies hörte, staunte er und befahl ihnen die Hände abzuhauen. Und gleich ergriffen sie die Heilige, banden dieselbe, legten sie auf den Amboß und ergriffen die Hackmesser, und schlugen und hackten die drei Diener auf die Hände der heiligen Photine siebenmal wechselnd los, und konnten nichts ausrichten, bis sie ganz kraftlos wie Tote niederfielen. Die Heilige aber wurde durch die Gnade Christi unversehrt bewahrt und betete und sprach: „Der Herr ist mein Helfer! Ich fürchte nicht, was kann ein Mensch mir tun!“

Da begann der Kaiser vor Staunen nachzudenken, wie er mit verschiedenen Listen die Heiligen quälen möchte. Und er ließ jene in einen tiefen Kerker abführen, die heilige Photine aber mit ihren fünf Schwestern befahl er in sein goldenes Kubulium (vermutlich cubiculum, Schlafgemach) $\mathrm{zu}$ führen und sieben Leuchter und einen Tisch vorzusetzen. Und er befahl seiner Tochter Domnina hineinzugehen und mit ihnen samt ihren Sklavinnen zu verweilen. Und er legte viele Schätze und goldene Zeichen (?) und Kleider und goldene Gürtel hin. Als aber die heilige Photine das Mädchen Domnina erblickte, sprach sie: „Sei gegrüßt, Braut Christi!“ Diese aber antwortete: „Sei gegrüßt auch du, meine Herrin, leuchtende Fackel Christi!" Und es wurde die Kaisertochter von den heiligen Frauen belehrt mit ihren 100 Sklavinnen, und sie tauften sich. Und [Photine] nannte die Kaisertochter Domnina Anthussa. Und gleich befahl die selige Anthussa all ihr Gold, und alles, was in ihrem goldenen Kubuklium war, den Armen zu verteilen durch die Hand der Stephanide, der Ältesten der Sklavinnen.

Als aber der Kaiser dies erfuhr, ergrimmte er und befahl drei Tage den Ofen $z u$ heizen und in denselben die heilige Photine und alle die mit ihr .waren, bis 3000 an der Zahl, Männer und Weiber, hineinzuwerfen. Nachdem dies geschehen war, weilten sie im Innern des Ofens drei Tage. Nach drei Tagen aber, in der Meinung, die Heiligen seien vom Feuer verzehrt worden, ließ er den Ofen aufmachen und ihre Gebeine in den Fluß werfen. Und nachdem aufgemacht worden war, fand man die Heiligen Gott lobend und preisend, und alle erschraken, da das Feuer

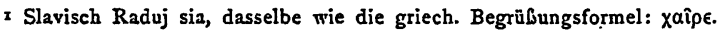


sie gar nicht berührt hatte. Und als alle Leute der römischen Stadt dieses berühmte Wunder hörten und sahen, wunderten sic sich und priesen Gott auch dicse.

Der Kaiser aber war Augenzeuge dieses Wunders und befahl ihnen ein giftiges Kraut zu geben. Es wurde ein Zauberer Lambadius gerufen und $\mathrm{cr}$ goß das Kraut ein und gab es zuerst der heiligen Photine. Sie aber nahm es an und sprach: „Du unreines, verzaubertes Kraut, es geziemte dir nicht uns auch nur zu berühren; doch damit jener, der dich gebraut hat, auch die Kraft Christi und meines Gottes erkenne, da will ich als erste von allen dies austrinken im Namen des einzig wahren Gottes und unseres Heilands Jesu Christi, und dann alle bei mir Anwesenden." Und nachdem alle getrunken hatten und unverschrt geblieben waren, sah dies der Zauberer und verwunderte sich und sah die Heilige an und sprach: „Ich habe noch ein künstliches Kraut, und wenn du davon kostest und nicht bald stirbst, so will auch ich an Christus, euren Gott, glauben." Und als das Kraut gebracht wurde lind alle davon kosteten und nichts Schlimmes erlitten, sammelte er alle seine Zauberbücher und übergab sie dem Feuer, glaubte an Christus, taufte sich und empfing den Namen Christodulos.

Als der Kaiser dies erfahren, befahl er ihn aus ihrer Mitte herauszugreifen und außerhalb der Stadt zu köpfen. Und so vollendete Christodulos vor allen anderen sein Martyrium, um Christi willen geköpft, und empfing die Krone und ging in den Himmel hinauf. Allen Heiligen aber mit der Großmärtyrerin Photine ließ der verruchte Nero die Adern aufschlitzen. Und als dies geschehen war und die Heiligen ihn verhöhnten und seine Götter verspotteten, befahl er, die Heiligen mit siedendem Blei und Schwefel zu tränken, sọvie ihnen solches auf die Schultern zu gielien. Und als dies geschah, riefen alle wie mit einem Munde: „Wir danken dir, Herr unser Gott, daß du unsere Herzen mit siedendem Blei betaut hast, wie die vor Sonnenhitze Lechzenden und Verbrannten!“ Nero verwunderte sich sehr und befahl die Heiligen aufzuhängen und sie gewaltig am ganzen Leib $z \mathrm{u}$ kratzen und mit brennenden Fackeln zu sengen. Als dies geschah und die Heiligen beteten und durch die göttliche Gnade fest blieben, ergrimmte der verruchte Nero und lieb siedendes Wasser mit starkem Essig vermischen und den Heiligen in die Nasenlöcher hineingießen. Als dies an den Heiligen vollzogen wurde, sprachen sie: „Es ist uns wie Meth und Honigseim." Und noch ärger ergrimmte der Tyrann und befahl, ihnen die Augen auszustechen und sie blind in einen dunklen und stinkenden Kerker zu werfen, der von 
giftigen Schlangen und Skorpionen voll war. Die Heiligen sangen Gott mit großer Stimme, und die im Kerker gehaltenen giftigen Tiere krepierten und der Kerker erfüllte sich mit einem unaussprechlichen Wohlgeruch und das Dunkel verwandelte sich in glänzendes Licht. Und da stand Christus unter den Heiligen und sprach zu ihnen: „Friede sei mit euch!" Und er nahm die Heilige [bei der Hand] und richtete sie auf und sprach: „Freuet euch, denn ich bin mit euch, freuet euch heut' und immerdar." Und von diesem Wort wurden ihre Augen sehend und sie sahen den Herrn. Und er segnete sie und sprach:,-, Seid mannhaft und stark!" und so stieg er zum Himmel auf. Von den Leibern der Heiligen fiel es wie Schuppen herab und sie wurden gesund, wie sie früher gewesen.

Der leidenschaftliche und gottverhaßte Nero aber ließ die Heiligen mehr als drei Jahre im Kerker venweilen, „bis sie - sagte er - in einem schlimmen Tode ihre Seelen verlieren." Nach drei Jahren aber hatte der Kaiser einen Sklaven aus seinem Palaste, welcher im Gefängnis gehalten wurde, und ließ diesen befreien. Und als die vom Kaiser [zu diesem Behuf] Geschickten die Heiligen gesund sahen, erzählten sie, die geblendeten Galiläer seien sehend und gesund, ihr Gefängnis so hell erleuchtet und lasse sehr viele Wohlgerüche emporsteigen, indem es sich in einen Ruhm Gottes und in ein heiliges Haus verwandelt hat, und in der Mitte des Kerkers sei ein siedendes Reichtum (offenbar wird eine wunderbare Springquelle gemeint), wo die Leute zusammenlaufen, sich von ihnen taufen lassen und an ihren Gott glauben. Nachdem der Kaiser dies gehört hatte, wunderte er sich, schickte und ließ sie vor sich führen und sprach: „Habe ich euch nicht mit kaiserlichem Gebot aufgetragen, Christum in der römischen Stadt nicht zu predigen? Wie wagt ihr so etwas im Kerker zu tun? Deswegen verfüge ich viele und große Qualen über euch.“

Diese sagten: „Tue, was du willst! Wir werden nicht unterlassen Christum als den wahren Gott und Schöpfer des IVeltalls zu predigen."

Deswegen von Grimm entbrennend, ließ er sie kopfunter kreuzigen und drei Tage lang ihre Körper mit Ochsénziemern peitschen ", „bis ihre Glieder - sagte er - auseinander fallen". Nachdem dies geschehen war, stellte er Wachen auf und ließ sie vier Tage lang hängend bewachen. Als aber am vierten Tag die Diener herbeikamen, um zu sehen, ob sie noch am Leben sind, und als sie sie hängend sahen, da

I In Slavischen: razčesyvati žylami, mit Adern durchkämmen. 
crblindeten sie. Ein Engel Gottes aber kam vom Himmel herab, band die Heiligen los, kübte sie und machte sie gesund. Dann erbarmte sich dic Heilige (scil. Photine) der geblendeten Diener und betete, und gleich wurden sie sehend, glaubten (an Christus), tauften sich im Namen Cliristi und folgten ihnen, nach.

Als der abscheuliche Nero dies hörte, ergrimmte er, der verdammte, und befahl, der heiligen Photine die Haut abzuschinden. Als dies geschah, sang dic Heilige: „Herr, du hast mich erprobt!" Als sie ihr die Haut abgeschunden hatten, warfen sie dieselbe in den Flulb und die Heilige stürzten sie in eine trockene Zisterne. Den Sebastian, Photinus und Josias aber ergriffen sie und schnitten ihnen die Schamteile samt den Waden ab und warfen sie den Hunden vor, schunden ihnen die Haut von den Köpfen ab und warfen sie in ein altes Bad hinein. Dann brachten sie die fünf Schwestern der heiligen Photine herbei, schnitten ihnen die Brüste $a b$ und schunden hernach auch ihnen die Haut ab. Als sie aber an die heilige Photis kamen, wollte sie sich von keinem ergreifen lassen, wie die andern, sondern stellte sich heldenhaft hin und rib selbst ihre Haut $a b$ und warf sie Nero ins Gesicht, so daß der Kaiser über ihre Mannhaftigkeit und Geduld sehr erstaunt war. Eine andere grimmige und tödliche Qual erdachte er für sie: in seinem Garten ließ er zivei Bäume mit Gewalt herumbiegen und die Heilige bei den Füßen daran binden, und als sie losgelassen wurden, zerrissen sie die Heilige, und sie übergab Gott ihre heilige und selige Seele. Dann ließ der Schlimme die übrigen mit dem Schwerte hinrichten. Die selige Photine aber holte er aus der Zisterne herauf und warf sie in den Kerker. Dort lebte sie noch 20 Tage. Dann lieb er sie vorführen und fragte, ob sie bereuen wolle und sich herbeilasse seinen Göttern ein Opfer darzubringen? Die Heilige aber spuckte ihm ins Gesicht, verspottete seine Eitelkeit und seine sinnlose Denkweise und sprach: „Du allerabscheulichster Blinder! Denkst du, ich sei so unverständig, du Lügner und Dummkopf, daß ich meinen Herrn verleugnen und deinen blinden Göttern opfern werdẹ?" Dies hörend, befahl der Kaiser, sie wieder in die Zisterne herabzuschleudern. Und als dies geschah, gab die Heilige ihre Seele an Gott auf, einen Kranz tragend und ewig jubilierend.

Es ist genug, diese lebhafte, dramatische Darstellung, gepaart mit der grauenhaftesten Phantastik nur einmal zu lesen, um den Gedanken an das Nachforschen des historischen oder geographischen Hintergrundes derselben lächerlich zu finden. Was hilft es uns, den Heerführer Viktor, 
welcher zu Neros Zeit mit Avaren kämpft und dann den Auftrag erhält, die Christen in Italien zu verfolgen, mit dem römischen Legionär Viktor zu identifizieren, welcher unter Vespasian in Palästina kämpfte, wie es die Bollandisten tun? Oder was helfen uns alle Nachforschungen nach jenem Dux Sebastian, welcher in unserer Erzählung offenbar als Kommandant einer Stadt, genannt Italien, gedacht wird? Wir sehen ja gleich, daß der ganze Hintergrund dieser Erzählung durchaus phantastisch ist, ebenso phantastisch, wie die in ihr dargestellten menschlichen Verhältnisse. Dieser Heerführer, welcher an der Straße sitzen soll, um gleich einem Straßenräuber vorïbergehende Christen nach ihrem Katechismus auszufragen und $\mathrm{zu}$ berauben, dieșe Photine, welche wie eine zweite Königin ron Saba mit einem ungeheuren Gefolge aus Karthago nach Rom zieht, um sich und die Ihrigen hier martern $z$ u lassen, dieser Nero, welcher der halsstarrigen Christin zu unbekanntem Zwecke gleich seine Schätze und seine Tochter vorsetzt, - alles dies zeigt deutlich, daß wir hier keine Spur historischer Reminiszenz, sondern nur ein pures Phantasiespiel vor uns haben, keine echte Legende, sondern das Bruchstück eines christlichen Romans im Geschmacke der Pseudoklementinen (sit venia verbo), oder der apokryphen Apostelgeschichten. Ebenso wie spätere Generationen diese phantastischen und im Rahmen der Phantastik gewisse sektiererische Doktrinen propagierenden Erzeugnisse für das katholische Publikum in der Weise adoptierten, daß sie das Gift der Doktrin mehr oder weniger gründlich entfernten und das phantastische Grund-

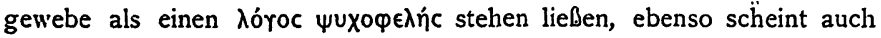
hier geschehen zu sein. $\mathrm{Dab}$ wir in unserer Erzählung nur ein von lehrhaften Bestandteilen ausgeweidetes Bruchstück eines Romans vor uns haben, braucht ja nicht speziell bewiesen zu werden. Die Situation zu Anfang der Erzählung ist ja gleich in mediis rebus; Dux Sebastian spricht mit Viktor wie mit einem alten Bekannten und sogar wie mit einem intimen Freund, dessen Mutter und Bruder er kennt. $\mathrm{Da}$ sie intime Freunde sind und trotzdem Sebastian noch ein Heide, läßt vermuten, daß auch Viktors Christentum nicht alten Datums ist und daß der gegenwärtigen Erzählung eine womöglich wunderbare Bekehrungsgeschichte vorangegangen sein muß. Über den Inhalt dieser Bekehrungsgeschichte finden wir eine Andeutung in der Rede der Photine vor Nero, wo sie auf des Kaisers Anfrage über ihren Namen antwortet: „Vom Christus, meinem Herrn, bin ich Photine genannt worden." Nun wissen wir aber von einer solchen, quasi durch Christum bewerkstelligten Namengebung gar nichts und es waren wahrscheinlich erst die späteren 
byzantinischen Legendenschreiber, welche die bereits auf den gegenwärtigen Zustand reduzicrte Erzählung auf Grund eben dieser Worte mit der Episode über die Samariterin im Johannesevangelium in Verbindung brachten, von der Hypothese ausgehend, Christus hätte, wenn je, so doch nur damals, in intimen Gespräch mit der Samariterin ihr auch einen solchen Namen gegeben haben. Doch es genuigt wieder, unsere Erzählung nur cinmal zu lesen, um sich zu übcrzeugen, da Photine mit jencr Samariterin gar nichts gemein hat, dab sie als eine reiche, angesehene karthagische Patrizierin auftritt, deren Soln ein römischer General ist und deren Erscheinen in Rom eine allgemeine Sensation hervorruft. Nur einmal scheint der Text auf ihren palästinischen Ursprung hinzudeuten, indem alle diese karthagischen Damen "Galiläerinnen" genannt werden. Doch auch dies reimt sich schlecht mit ihrem Samaritanertum und erklärt sich weit besser als der verächtliche Name der Christen überhaupt, wie wir ihn aus zahlreichen Martyrien nach der diokletianischen Christenverfolgung kennen.

So wie sie da ist, scheint diese Passio S. Photinae nur eine zwecklose Anhäufung der horrenden Greuelszenen zu sein, ein Zeugnis für die blutgierige Phantasie und den schlechten Geschmack ihres Verfassers. Erst wenn wir sie als ein Epitome aus dem verlorenen Ganzen betrachten, wenn wir auf ihr Spuren und Narben der einst am Prototyp vorgenommenen Operation verfolgen und dieselben mit anderen derartigen uns wohl bekannten Zeugnissen in Verbindung betrachten, gewinnt sie für uns eine gewisse religions- und literaturgeschichtliche Bedeutung. Da möge nun vor allem an die auffallende Sucht der Namenänderung hingewiesen werden, welche hier vorherrscht und als deren Urheber Christus selbst dargestellt wird. Bemerkenswert ist auch die Tendenz, alle Namen mit den Begriffen: Licht, Sonnenaufgang in Verbindung zu bringen; für den Kirchenhistoriker wird dies vielleicht ein Fingerzeig sein, in welcher häretischen Sphäre er den Ursprung unserer Erzählung zu suchen hätte. $\mathrm{Zu}$ den kulturgeschichtlich interessanten Erscheinungen dieser Erzählung gehört die Erwähnung der durch ein Weib vorgenommenen Taufe (in der Episode mit der Tochter Neros, welche in der Episode mit dem Zauberer Lambadius ihre nicht so deutlich aus gesprochene Doublette hat), welche uns an die Acta Pauli et Theclae mit. der sich selbst und ihre getreue Löwin taufenden Jungfrau erinnert. Wenn nicht gnostische Doktrin, wovon freilich fast keine Spuren übriggeblieben sind, so herrscht doch die gnostische Phantasie, wie sie Lipsius in seinem Werke über die apokryphen Apostelgeschichten so gut charak- 
terisiert hat, auch in dieser Erzählung vor. Häufige Erscheinungen der Engel und besonders Christi in Person, statt sprechender Tiere solche, welche bei der Berührung der Heiligen sterben, die orientalischen Martern, wie die Hautabschindung (wie beim heiligen Thomas und Bartholomäus), wobei der Geschundene dann noch tage- und jahrelang am Leben bleibt, die Augenausbohrung, das wunderbare Anwachsen zerstörter Glieder, die übernatürliche Widerstandskraft gegen die Qualen, alles dies führt uns in die wohlbekannte Atmosphäre jener sektiererischen Phantasiegebilde, welche wie eine dichte Wolke die ersten vier Jahrhunderte der Geschichte des Christentums umflorén und ihrer ganzen weiteren Entıvicklung ihren unverlöschilichen Stempel aufdrückten. 